\title{
An accelerating handover process scheme for IEEE 802.16j multi-hop relay networks
}

\author{
Jau-Yang Chang
}

\begin{abstract}
IEEE 802.16j multi-hop relay network systems provide the mobile wireless communication environment. In such network systems, the handover scanning procedure allows a mobile station (MS) to obtain the information about the handover target base stations (BSs) or relay stations (RSs). The network systems need more time to negotiate the association parameters and to handle scanning the BSs and the RSs when the number of BS and RS increases. It results in more overhead for the handover scanning procedure. In order to accelerate the handover process and reduce the transmission interruption, efficient handover scanning procedure schemes and corresponding algorithms must be developed and designed. A novel relative angle computing algorithm is proposed in this article to accelerate the handover process by taking into account the moving behavior of the MS, and the distances among the MS, the RS, and the BS. The main idea of this algorithm is to reduce the management information overhead and to estimate the potential moving path of the MS in the wireless mobile communication networks. By using the proposed scheme, we eliminate the unnecessary associations and scanning intervals, and reduce the handover scanning procedure efficiently. Simulation result demonstrate the superior performance of our proposed scheme and its ability to strike the appropriate performance in the handover overhead and the message delay for IEEE 802.16j multi-hop relay network systems.
\end{abstract}

Keywords: Base station, Handover, IEEE 802.16j, Message delay, Mobile station, Relay station

\section{Introduction}

IEEE 802.16 Broadband Wireless Access Systems, also refereed as WiMax (Worldwide Interoperability of Microwave Access), is designed to evolve as a suite of air interface for fixed, portable, and mobile wireless access systems. This standard is a promising technology to support high transmission rate and predefined quality-of-service (QoS) framework in the broadband wireless networks. This technology can provide a cost-effective broadband access solution to use for connecting local area networks to the Internet and to support mobile applications such as fourth generation mobile systems [1].

IEEE 802.16j multi-hop relay network specifies a system for combined fixed and mobile broadband wireless access environments [2]. This standard provides a mobile environment for the mobile stations (MSs). In such system, the MSs can send their data directly to the base station (BS) or use some relay stations (RSs) to relay

Correspondence: jychang@nfu.edu.tw

Department of Computer Science and Information Engineering, National Formosa University, Hu-Wei, Yun-Lin, Taiwan their data to the BS. The coverage of the system can be enhanced and more MSs can be accepted in the system. Because the distance between a pair of communicating station is shortened, it reduces the power consumption, path loss, and enables communication at higher data rate. Figure 1 illustrates an example of IEEE 802.16j multi-hop relay system model. The places of RSs depend on realistic environments such as the shadow of buildings, valley between the buildings, and the coverage extension at the cell edges. However, the additional handovers occur in a multi-hop cellular network between the BS and the RSs or between two RSs. The network systems need more time to negotiate association parameters and to handle scanning BSs and RSs when the number of BS and RS increases. It results in more overhead for the handover scanning procedure. The long interruption of hard handover (HHO) is serious for the packet delay sensitive applications. In order to accelerate the handover process and reduce the transmission interruption, efficient handover scanning procedure schemes must be developed in an IEEE 802.16j multi-hop relay network system.

\section{穴}

(c) 2013 Chang; licensee Springer. This is an Open Access article distributed under the terms of the Creative Commons Attribution License (http://creativecommons.org/licenses/by/2.0), which permits unrestricted use, distribution, and reproduction in any medium, provided the original work is properly cited. 


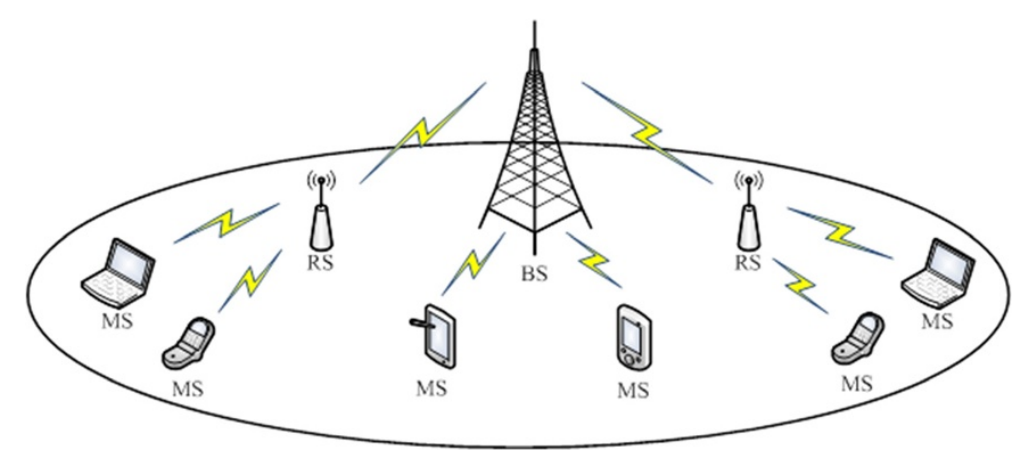

Figure 1 IEEE 802.16j multi-hop relay system model.

Several researches on the relay issues have been conducted in IEEE 802.16j multi-hop relay network systems. With respect to the relay selection issues, Ann et al. [3] propose a path selection method considering the link available bandwidth, signal-to-noise ratio (SNR), and hop count in non-transparent mode of IEEE 802.16j. This scheme only suggests a suitable path for the new RSs and it does not consider the mobile environment for the MSs. Ge et al. [4,5] analyze the relay selection in IEEE 802.16j multi-hop relay vehicular networks. An analytical model is developed in this article for locating and selecting the RS based on the locations of MSs. These two articles only consider a transport system with the highway mobility pattern. An effective path selection metric for IEEE 802.16j multi-hop relay networks is proposed in [6] to improve the network throughput. The authors use an effective radio resource index to calculate the cost function and determine the relay path between the BS and the MS. Shih et al. [7] propose a high spectral efficiency and load-aware metric for the path selection in IEEE 802.16j multi-hop relay networks. A comprising function is developed in this article to formulate the path cost to evaluate the possible relay paths and improve the network throughput. Previous schemes described above do not take into account the problem in the handover process with respect to QoS guarantees for the MSs in an IEEE 802.16j multihop relay network. These previously developed schemes ignore the discussion of the handover overhead and message delay for an MS. They only emphasize the network throughput enhancement.

Several handover techniques have been proposed to support QoS provisioning in IEEE 802.16-based systems. With regard to the handover issues in IEEE 802.16e network systems, analytical models have been proposed in [8-13], which have been validated to some extent through simulations. Nevertheless, only a few research articles have studied handover issues in IEEE 802.16j network systems. A reducing inter-cell handover events (RIHE) based on cell id information in multi-hop relay system is proposed in [14] to decrease handover signaling overhead, latency, and unnecessary handovers. The authors propose a handover method that reduces intercell handover but increases intra-cell handover events by modifying the BSID format into the hierarchical BS/RS ID. Becvar et al. [15] propose an optimal handover scanning procedure (OHSP) in IEEE 802.16j network systems to reduce the management information overhead and to maximize the user data throughput. The modification of scanning procedure is based on the uplink direction by adding identification addresses of all recommended stations for scanning into one scanning request message. In [16], this article presents handover schemes in multihop cellular networks (HSMCN) where RSs are located either inside a cell or on the boundary between two adjacent cells. By deploying RSs on the boundary between two adjacent cells, the service-interruption time caused by inter-cell handover is reduced. Yun et al. [17] propose a fast handover scheme (FHS) to reduce the handover signaling cost and to decrease handover delay by applying fast handovers for mobile IPv6 (FMIPv6) to IEEE 802.16j networks, which determines the cross-subnet handover in advance through the interaction of MAC layer messages. Sultan et al. [18] perform simulation study of three handover techniques (SSTHT) within the IEEE 802.16j standards and prove that the macro diversity handover (MDHO) outperforms the $\mathrm{HHO}$ and fast base station switching. MDHO is the process by which an MS maintains connection with two or more access stations called a diversity set. In [19], a topology-aware macro diversity handover technique (TMDHT) is proposed to improve the conventional MDHO.

These previously developed schemes [15-17] described above do not take into account the moving situation of MSs. Due to users' mobility and insufficient bandwidth in the mobile wireless networks, it is important to provide QoS guarantees such as the message delay for an MS in IEEE 802.16j systems. From the point of view of mobile user, the message delay is obviously undesirable and very inconvenient, so the mobile users are not 
tolerant of this problem. Unsatisfied mobile users may change to the competing system. Hence, it is important to accelerate the handover process in IEEE 802.16j multi-hop relay networks. The message delay becomes the most significant QoS factor in such networks. Therefore, the performance parameters of interest in this article are the handover overhead and the message delay.

Motivated by the above discussion, in order to reduce the handover scanning procedure efficiently, it is important to consider the users' mobility. There are several parameters that directly affect the users' mobility: geographic topography (e.g., roads, streets, and lanes), transportation way (e.g., railway, freeway, and highway), common and personal habits (e.g., go to work, go back home, and go shopping). In most cases, the trajectories of users are foreseeable. In the proposed scheme, we take into account a more realistic environment of random movement of MSs in IEEE 802.16j multi-hop relay network systems. We propose a novel relative angle computing algorithm (RACA) in this article to estimate the potential moving path of the MS by taking into account the moving behavior of the MS and the distances among the MS, the RS, and the BS. The global positioning system (GPS) is not employed in our proposed scheme because the mobile devices do not necessarily have the functions of GPS. Based on the existing network conditions, the proposed scheme makes an adaptive decision. Thus, our proposed scheme has the ability to provide more efficient control over network condition fluctuations. In addition, our system takes into account environments encountered in practice, and to test its response different number of RSs and MSs that are simulated and discussed in this article. This is important for evaluating the handover overhead and message delay performance. By using the proposed scheme, we eliminate the unnecessary associations and scanning intervals, and reduce the handover scanning procedure efficiently, while providing better QoS for mobile users in an IEEE 802.16j multi-hop relay network system. Table 1 summarizes a comparison of the handover methods proposed in the IEEE 802.16j systems.

The rest of this article is organized as follows. In Section 2, we present the system model of IEEE 802.16j multi-hop relay networks. In Section 3, we illustrate the proposed scheme in detail. In Section 4, we present our simulation model and analyze the comparative evaluation results of the proposed scheme through the simulations. Finally, some conclusions are given in Section 5.

\section{System model}

In this article, we consider a mobile wireless network with a multi-hop relay network infrastructure. The system infrastructure is composed of BSs, RSs, and MSs. IEEE 802.16j multi-hop relay network system allows one or more RSs to be deployed between the BSs and the MSs for extending the coverage and enhancing the performance of the network. The BS is directly connected to the wired backhaul and is equipped with sufficient intelligence that handles all the routing and connection issues in the cell. The RS is not directly connected to the wired backhaul and is responsible for relaying data between the BS and the MS. An RS can be deployed on the tower and have sufficient power to transmit the signal. The function of the RS is considerably less complex than that of the BS. Hence, the RS deployment cost is also expected to be much lower compared with that of the BS. The MS will directly communicate with a BS or through an RS. When the MS is far away the BS or the signal strength is poor between the BS and the MS, the MS can switch to the RS and can relay its data to the BS. In this article, we focus on handover scanning overhead analysis based on the random mobility environment, where the MSs can randomly move in the wireless network systems. Figure 2 shows the handover in IEEE 802.16j multi-hop relay network systems, where the handover scenarios are classified into intracell handover and intercell handover with the HHO technique [16]. In Figure 2, MS1 and MS3 result in the intracell handover, while MS2, MS4, and MS5 result in the intercell handover.

When an MS roams in the multi-hop relay networks, it must obtain the information about the neighbor BSs or RSs to prepare for switching from the current serving $\mathrm{BS} / \mathrm{RS}$ to the new BS/RS. As the signal strength is poor between the serving BS/RS and the MS, the MS must seek a suitable neighbor BS or RS to perform the handover. Figure 3 shows the handover signaling in IEEE

Table 1 Comparison of the handover methods proposed in the IEEE 802.16j systems

\begin{tabular}{ccccccc}
\hline Method & Handover technique & RS Location & $\begin{array}{c}\text { Number of RSs } \\
\text { (per cell) }\end{array}$ & $\begin{array}{c}\text { Number } \\
\text { of hops }\end{array}$ & $\begin{array}{c}\text { Number of } \\
\text { connection stations }\end{array}$ & $\begin{array}{c}\text { Moving situation } \\
\text { of MSs }\end{array}$ \\
\hline RIHE [14] & HHO & Fixed & 6 & 1 & 1 & Yes \\
OHSP [15] & HHO & Random & $0-50$ & $\geq 1$ & 1 & No \\
HSMCN [16] and FHS [17] & HHO & Fixed & 6 & 1 & 1 & No \\
SSTHT [18] and TMDHT [19] & MDHO & Fixed & 6 & 1 & 1 & Yes \\
Proposed RACA & HHO & Random & $10-50$ & 1 & & Yes \\
\hline
\end{tabular}




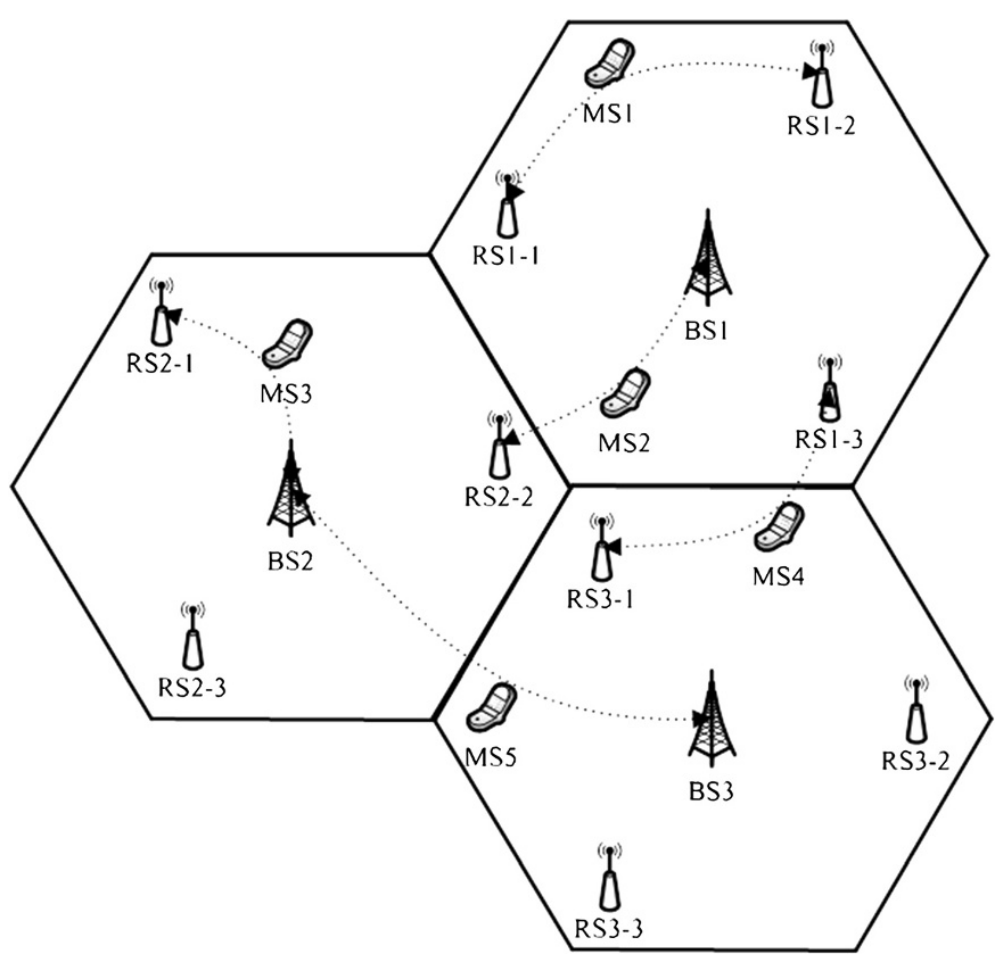

Figure 2 Handover in IEEE 802.16j multi-hop relay network systems.

802.16j multi-hop relay network systems [16]. The handover procedure includes two phases: received-signal -strength (RSS) measurement and handover decision phases. In the RSS measurement phase, the MS scans the suitably neighbor BS/RS to be a target BS/RS for handover by using the Measurement_REQ (MOB_SCNREQ) and Measurement_RSP (MOB_SCN-RSP) messages. An MS cannot receive data packets from the serving BS/RS during the RSS measurement for the neighbor BSs and RSs. The RSS measurement overhead increases because an MS should measure the RSS of the neighbor BSs and RSs. Therefore, the network systems need more time to handle the scanning BSs and RSs when the number of BS and RS increases. It results in more overhead in the handover scanning procedure. The message delay caused by handover can be increased in IEEE 802.16j multi-hop relay network systems. The QoS of the connection between the BS/RS and the MS is affected by the handover scanning procedure. The handover decision is based on the RSS measurement result. When the serving BS receives the RSS_Report message from the MS, it determines the handover execution. The MS will switch to the new BS/RS where the signal strength is the best between the new BS/RS and the MS. However, these networks have the inherent problem of rapid handovers due to the random movement of MSs. In addition, the moving direction of the MS does not necessarily go forward to the new BS/RS with the best signal strength in the future. The additional handover occur between the BS and the RSs or between two different RSs. It results in serious ping-pong problems and increases signaling overhead. Hence, the handover procedure must be improved in IEEE 802.16j multi-hop relay network systems. This article focuses on the RSS measurement phase to reduce the unnecessary associations and scanning intervals.

IEEE 802.16j uses an adaptive modulation coding scheme (MCS) for allocating different data transmission rates to different channel conditions. The received SNR is divided into several non-overlapping regions. Figure 4 shows the seven MCS in IEEE 802.16j network systems. Table 2 illustrates a transmission model that is the order of magnitudes of receiver SNR, data rate, and distance assumptions for different MCSs in the literature [1-10]. The distance between two communicating nodes changes with the time. Therefore, the receiver SNR also changes. When the SNR decreases at the receiver, the sender will adopt a lower order transmission mode to transmit at a lower achievable rate of the link. Similarly, as the SNR gets better, the sender switches its transmission mode to a higher order modulation for selecting the channel condition. Hence, the distance between two communicating nodes plays a major role in choosing the link transmission rate. The SNR value implies the 


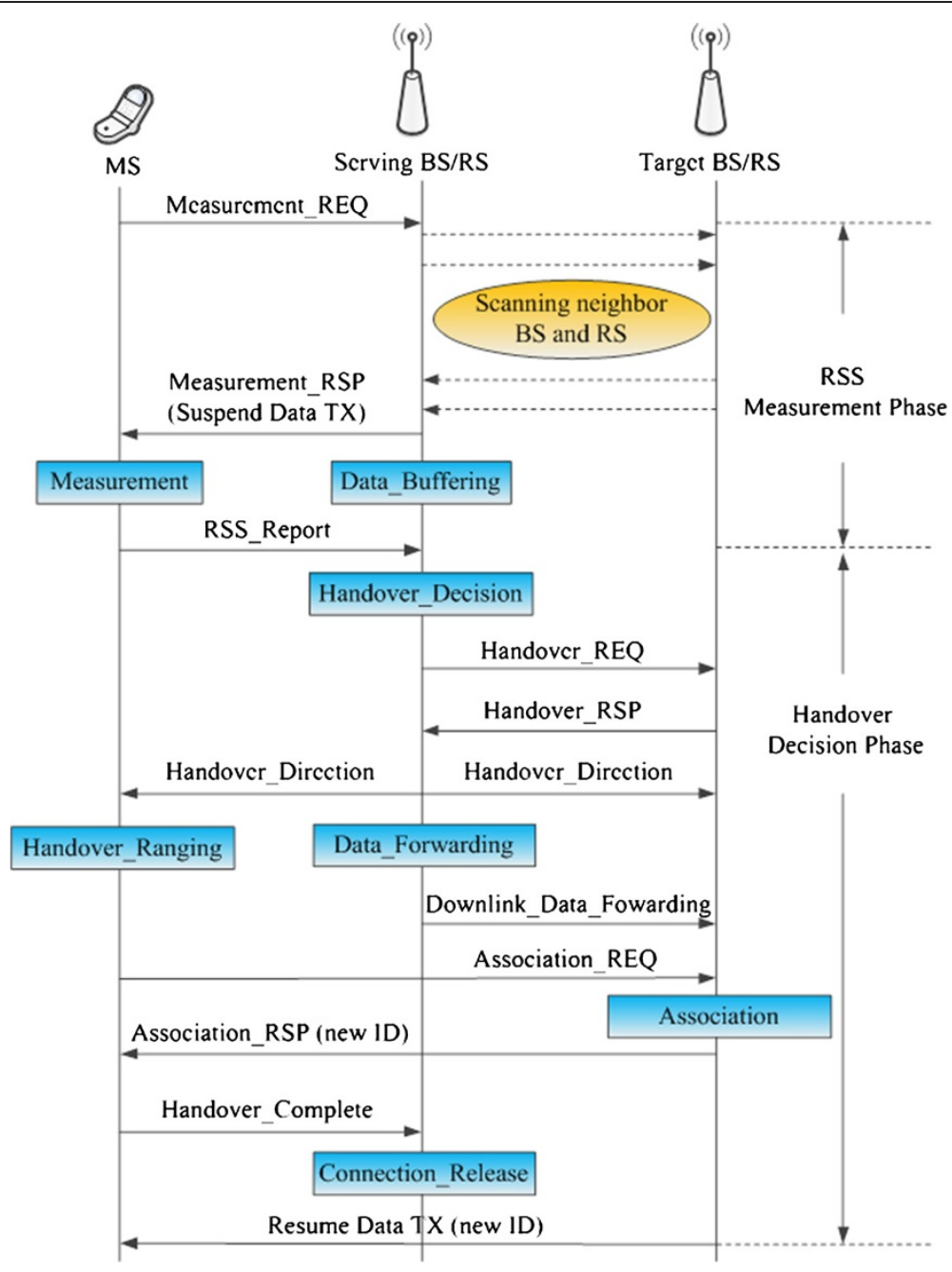

Figure 3 Handover signaling in IEEE 802.16j multi-hop relay network systems.

distance from the BS/RS to the MS [1-10]. According to the free space propagation model, the SNR of each link can be calculated by

$$
\mathrm{SNR}=10 \log _{10}\left(\frac{P_{t}}{P_{n}}\left(\frac{c}{4 \pi f d}\right)^{2}\right),
$$

where $P_{t}$ is the transmission power, $P_{n}$ is the thermal noise power, $f$ is the center frequency, $c$ is the speed of light, and $d$ is the distance between two communication nodes. The SNR value can be obtained in the RSS measurement phase in order to determine the handover execution. The distance between two communication nodes can be calculated based on the SNR value.

\section{Proposed RACA scheme}

In order to accelerate the handover process and reduce the transmission interruption, efficient handover scanning procedure schemes and corresponding algorithms must be developed. Based on the moving behavior of the MS, and the distances among the MS, the RS, and the BS, we propose a novel RACA to accelerate the handover process. The main idea of this algorithm is to reduce the management information overhead and to estimate the potential moving path of the MS in the wireless mobile communication networks. Figure 5 shows the example of possible moving path of the MS, where the distance between the RS and the MS is the same in positions $\mathrm{A}, \mathrm{B}$, and $\mathrm{C}$. It is clear that the moving direction of MS is hard to estimate when we only consider the distance (i.e., signal strength) between the MS and the BS/RS. In order to accurately estimate the moving direction of the MS, some angles are calculated according to the distances among the MS, the RS, and the BS. In Figure 5, when the MS moves from position A to position $B$, we can observe that $\theta_{3}$ is larger than $\theta_{1}$. In other words, when the MS moves from position A to position $C, \theta_{2}$ is smaller than $\theta_{1}$. Based on the changes 


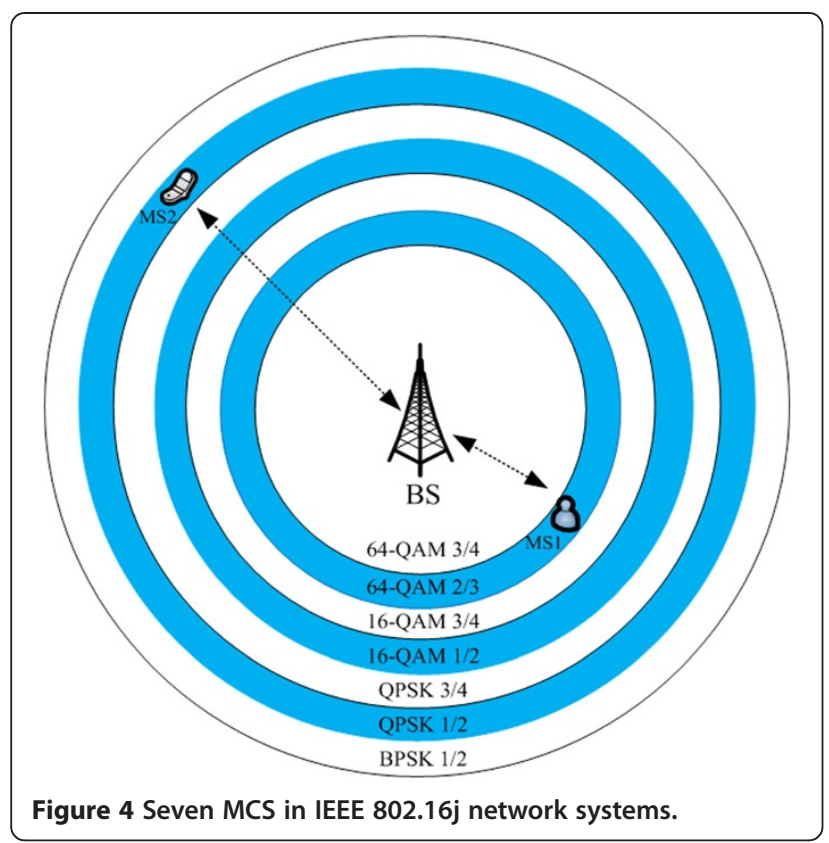

of angle, distance, and moving speed of the MS, the potential moving path of the MS can be estimated.

In the proposed scheme, we assume that the BS periodically calculates the distances among the MS, the RS, and the BS for each MS according to the signal strength and antenna gain [3-6,10-12]. We assume that an MS roams in coverage areas of RSs and the number of the RSs is equal to $r$. Let $d_{\text {SBRS,RS }}$ be the distance between the serving BS/RS and the RS $n d_{\mathrm{SBRS}, \mathrm{MS}}$ be the distance between the serving BS/RS and the MS, and $d_{\mathrm{MS}, \mathrm{RS}}$ be the distance between the MS and the $\mathrm{RS}_{r}$. Let $\theta_{r}$ be the angle formed by $d_{\mathrm{SBRS}, \mathrm{MS}}$ and $d_{\mathrm{MS}, \mathrm{RS}}$. According to the cosine theorem, $\theta_{r}$ can be calculated by

$$
\theta_{r}=\cos ^{-1}\left(\frac{d_{\mathrm{SBRS}, \mathrm{MS}}^{2}+d_{\mathrm{MS}, \mathrm{RS} r}^{2}-d_{\mathrm{SBRS}_{\mathrm{RSS}}}^{2}}{2 \times d_{\mathrm{SBRS}, \mathrm{MS}} \times d_{\mathrm{MS}, \mathrm{RS} r}}\right) .
$$

Figure 6 shows the example of the angles among the MS, the RSs, and the serving BS/RS, where the number of RSs is equal to three. The distance between the MS and BS/RS changes with the time. Therefore, the angles

Table 2 Transmission model

\begin{tabular}{lcccc}
\hline Modulation & Coding rate & $\begin{array}{c}\text { Receiver } \\
\text { SNR }(\mathbf{d B})\end{array}$ & $\begin{array}{c}\text { Data } \\
\text { rate }(\mathbf{M b p s})\end{array}$ & $\begin{array}{c}\text { Distance } \\
(\mathbf{k m})\end{array}$ \\
\hline BPSK & $1 / 2$ & 3.0 & 1.269 & 3.2 \\
QPSK & $1 / 2$ & 6.0 & 2.538 & 2.7 \\
QPSK & $3 / 4$ & 8.5 & 3.816 & 2.5 \\
16-QAM & $1 / 2$ & 11.5 & 5.085 & 1.9 \\
16-QAM & $3 / 4$ & 15.0 & 7.623 & 1.7 \\
64-QAM & $2 / 3$ & 19.0 & 10.161 & 1.3 \\
64-QAM & $3 / 4$ & 21.0 & 11.439 & 1.2 \\
\hline
\end{tabular}

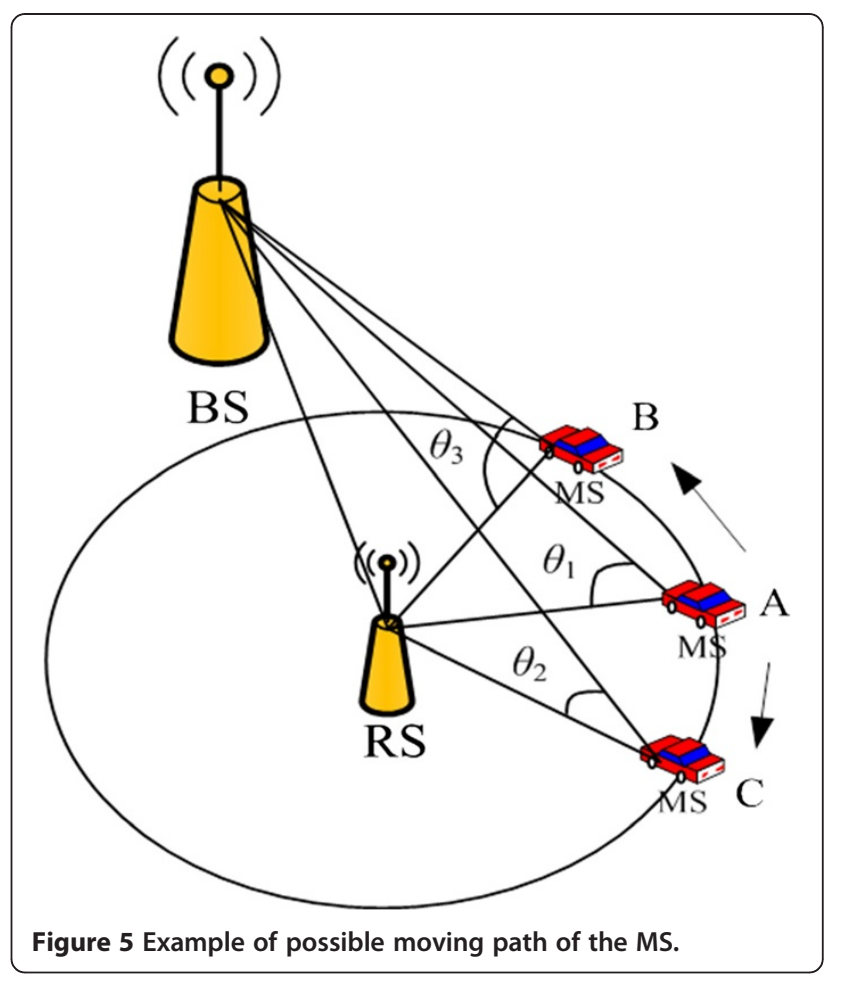

also changes. Figure 7 shows the example of the new angles among the MS, the RSs, and the serving BS/RS when the MS moves.

The moving speed of the MS plays an important role in the handover process. An MS with the high moving speed is easy to result in rapid handovers when the number of RS increases. Hence, we take into account the moving speed of the MS in the proposed algorithm. Let $\theta_{m, t_{n-1}}$ and $\theta_{m, t_{n}}$, respectively, be the angles formed by $d_{\mathrm{SBRS}, \mathrm{RS}}$ and $d_{\mathrm{MS}, \mathrm{RS}}$ at time $t_{n-1}$ and time $t_{n}$, which can be calculated by using the cosine theorem. Let $\Delta s$ be the moving distance of the MS from time $t_{n-1}$ to time $t_{n}$, which can be calculated by

$$
\Delta s=\sqrt{d_{\mathrm{MS}, \mathrm{RS}, t_{n-1}}^{2}+d_{\mathrm{MS}, \mathrm{RS}, t_{n}}^{2}-2 \times d_{\mathrm{MS}, \mathrm{RS}, t_{n-1}} \times d_{\mathrm{MS}, \mathrm{RS}, t_{n}} \times \cos \theta_{x}},
$$

where $\theta_{x}=\left|\theta_{m, t_{n}}-\theta_{m, t_{n-1}}\right|, d_{\mathrm{MS}, \mathrm{RS}, t_{n-1}}$, and $d_{\mathrm{MS}, \mathrm{RS}, t_{n}}$ are the distances between the MS and the RS at time $t_{n-1}$ and time $t_{n}$, respectively. Figure 8 shows the example of moving distance of the MS. Let $v_{\mathrm{MS}, t_{n}}$ be the moving speed of the MS at time $t_{n}$, which can be calculated by

$$
v_{\mathrm{MS}, t_{n}}=\frac{\Delta s}{t_{n}-t_{n-1}}
$$

A measurement function is designed in the proposed algorithm to check the unnecessary target BS/RS. Let $\theta_{r, t_{n-1}}$ and $\theta_{r, t_{n}}$, respectively, be the angles formed by $d_{\mathrm{SBRS}, \mathrm{MS}}$ 


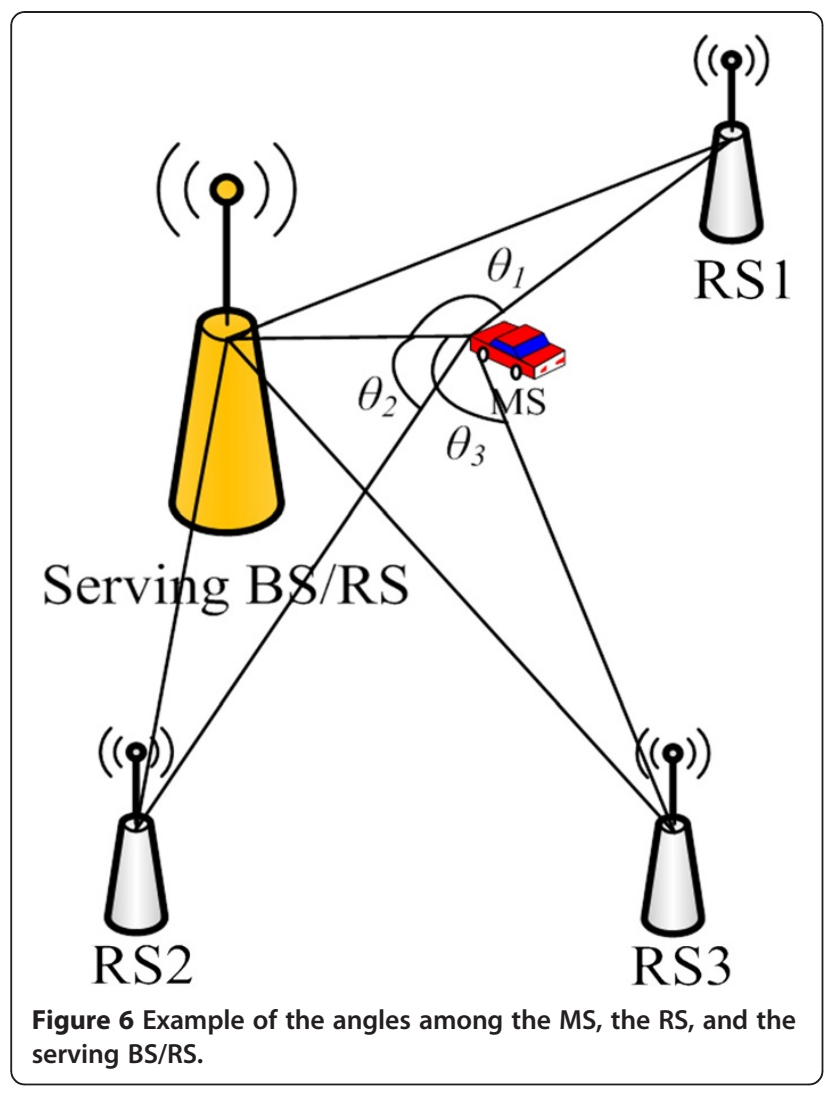

and $d_{\mathrm{MS}, \mathrm{RS}}$ at time $t_{n-1}$ and time $t_{n}$. Let $\alpha_{\mathrm{RS}_{\mathrm{r}}, t_{n}}$ denote the variation in angle formed by $d_{\mathrm{SBRS}, \mathrm{MS}}$ and $d_{\mathrm{MS}, \mathrm{RS}}$ from time $t_{n-1}$ to time $t_{n}$, which can be calculated by

$$
\alpha_{\mathrm{RS}}, t_{n}=\frac{\theta_{r, t_{n}}}{\theta_{r, t_{n-1}}}
$$

when $\alpha_{\mathrm{RS}_{r}, t_{n}}<1$, it means that the MS goes away the $\mathrm{RS}_{r}$ and the serving BS/RS. In contrast, when $\alpha_{\mathrm{RS}_{r}, t_{n}}>1$, it means that the MS goes forward to the $\mathrm{RS}_{r}$ or the serving BS/RS. According to Figures 6 and 7, the possible moving direction of the MS can be observed by using the parameter $\alpha_{\mathrm{RS}_{r}, t_{n}}$. However, the variation in angle formed by $d_{\mathrm{SBRS}, \mathrm{MS}}$ and $d_{\mathrm{MS}, \mathrm{RS}}$ can only observe the possible moving direction of the MS. It is hard to know whether the MS goes forward to the RS or not. This is because that the MS may go forward to the serving BS/RS when $\alpha_{\mathrm{RS}_{r}, t_{n}}>1$. Hence, the distance parameter must be taken into account. Let $\beta_{\mathrm{RS}_{r}, t_{n}}$ denote the variation in distance between the MS and the $\mathrm{RS}_{r}$ from time $t_{n-1}$ to time $t_{n}$, which can be calculated by

$$
\beta_{\mathrm{RS}_{r}, t_{n}}=\frac{d_{\mathrm{MS}, \mathrm{RS}_{r}, t_{n}}}{d_{\mathrm{MS}, \mathrm{RS} r}, t_{n-1}}
$$

when $\beta_{\mathrm{RS}_{r}, t_{n}}<1$, it means that the MS is gradually close to the $\mathrm{RS}_{r}$. In contrast, when $\beta_{\mathrm{RS}_{r}, t_{n}} \geq 1$, it means that the

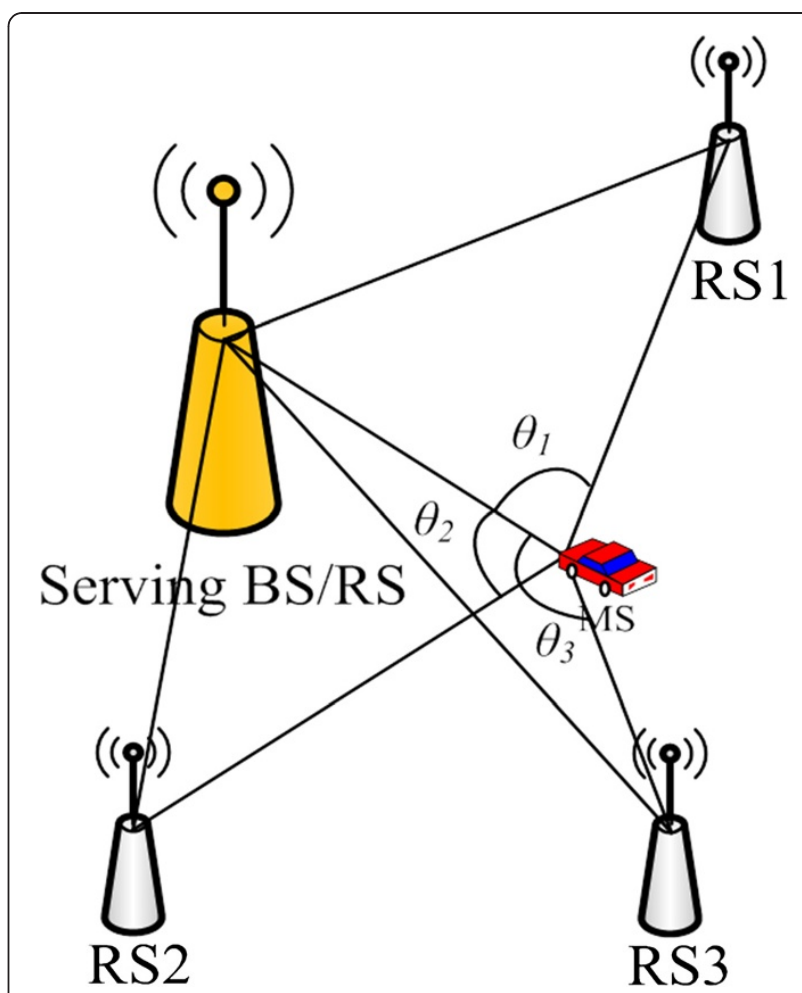

Figure 7 Example of the new angles among the MS, the RS, and the serving BS/RS.

MS goes away the $\mathrm{RS}_{r}$. Let $\omega_{\mathrm{RS}_{r}, t_{n}}$ be the measurement function of the $\mathrm{RS}_{r}$ for the MS at time $t_{n}$, which can be expressed by

$$
\omega_{\mathrm{RS}_{r}, t_{n}}=\frac{\sum_{t=t_{0}}^{t_{n}} \alpha_{\mathrm{RS}_{r}, t}}{\sum_{t=t_{0}}^{t_{n}} \beta_{\mathrm{RS}_{r}, t}}+\varepsilon_{t_{n}},
$$

where $t$ is the time measured from $t_{0}$ to $t_{n}$ after each handover and $\varepsilon_{t_{n}}$ is given by the following expression:

$$
\varepsilon_{\mathrm{RS}_{r}, t_{n}}=\left\{\begin{array}{cc}
\delta_{\mathrm{MS}, t_{n}} & \text { if } \alpha_{\mathrm{RS}_{r}, t_{n}}>1 \text { and } \beta_{\mathrm{RS}_{r}, t_{n}}<1 \\
-\delta_{\mathrm{MS}, t_{n}} & \text { if } \alpha_{\mathrm{RS}_{r}, t_{n}}<1 \text { and } \beta_{\mathrm{RS}_{r}, t_{n}}>1 \\
0 & \text { if } \alpha_{\mathrm{RS}_{r}, t_{n}}=1 \text { and } \beta_{\mathrm{RS}_{r}, t_{n}}=1
\end{array}\right.
$$

where $\delta_{\mathrm{MS}, t_{n}}$ is varying in velocity from time $t_{n-1}$ to time $t_{n}$, which can be calculated by

$$
\delta_{\mathrm{MS}, t_{n}}=\frac{v_{\mathrm{MS}, t_{n}}-v_{\mathrm{MS}, t_{n-1}}}{v_{\mathrm{MS}, t_{n-1}}}
$$

We employ $\varepsilon_{t_{n}}$ as a speed parameter to adjust the measurement function $\omega_{\mathrm{RS}_{r}, t_{n}}$. When $\alpha_{\mathrm{RS}_{r}, t_{n}}>1$ and $\beta_{\mathrm{RS}_{r}, t_{n}}<1$, $\varepsilon_{t_{n}}$ is equal to $\delta_{\mathrm{MS}, t_{n}}$. It means that the MS goes forward to the $\mathrm{RS}_{r}$ with a high opportunity. In contrast when $\alpha_{\mathrm{RS}_{r}, t_{n}}<1$ and $\beta_{\mathrm{RS}_{r}, t_{n}}>1, \quad \varepsilon_{t_{n}}$ is equal to $-\delta_{\mathrm{MS}, t_{n}}$. It 


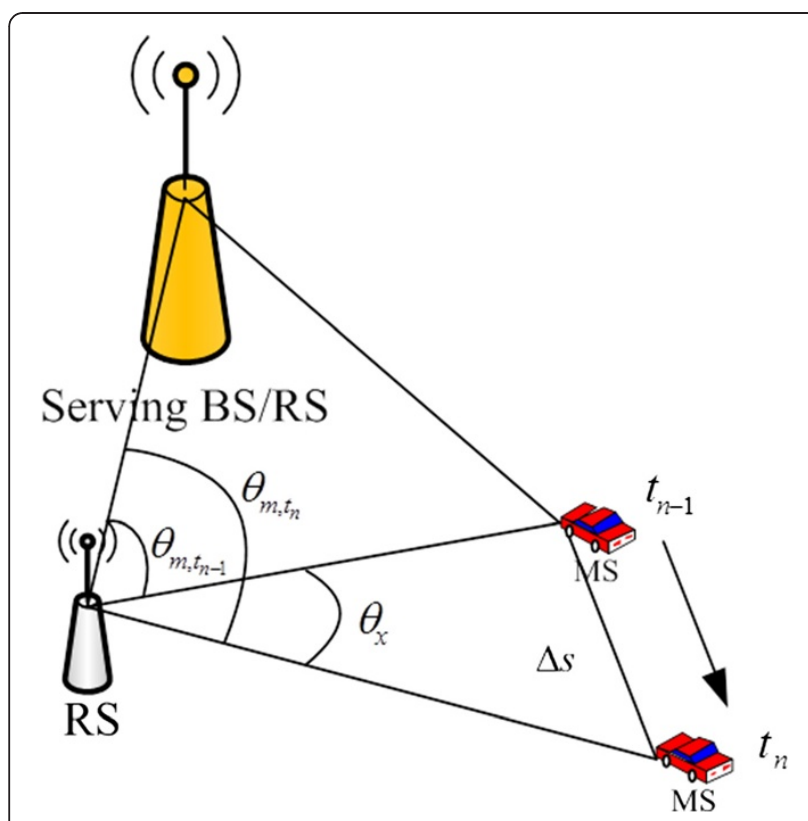

Figure 8 Example of moving distance of the MS.

means that the MS goes forward to the $\mathrm{RS}_{r}$ with a low opportunity.

An initial fully scanning process is required after the handover in order to re-calculate the measurement function $\omega_{\mathrm{RS}_{r}, t_{n}}$ in our proposed scheme. We employ the measurement function $\omega_{\mathrm{RS}_{\mathrm{r}}, t_{n}}$ to select the recommended RS for the MS. The recommended RS is an RS with the largest $\omega_{\mathrm{RS}_{r}, t_{n}}$. The recommended RS will become the target RS in the next handover. Hence, only one RS is required to scan and measure during the RSS measurement phase in the handover process. It can reduce the management information overhead and can eliminate the unnecessary associations and scanning intervals. Therefore, the handover process can be accelerated. Figure 9 shows the example of the recommended RS for the MS by using the measurement function of our proposed scheme according to the Figures 6 and 7. In addition, due to the user's mobility, an MS may slow down or stop to go to other direction. Hence, the speed parameter $\varepsilon_{t_{n}}$ is employed to adjust the measurement function $\omega_{\mathrm{RS}_{r}, t_{n}}$. When the MS slows down, $v_{\mathrm{MS}, t_{n}}$ is decreased. Hence, $\omega_{\mathrm{RS}_{r}, t_{n}}$ is also decreased and a fully scanning process is required in order to accurately reestimate the possible moving direction of the MS.

Due to an MS roams in the multi-hop relay networks, it must obtain the information about the neighbor BSs or RSs to prepare for switching from the current serving $\mathrm{BS} / \mathrm{RS}$ to the new BS/RS. As the signal strength is poor between the serving BS/RS and the MS, the MS must seek a suitable neighbor BS or RS to perform the handover. Hence, the BS periodically calculates the SNR level

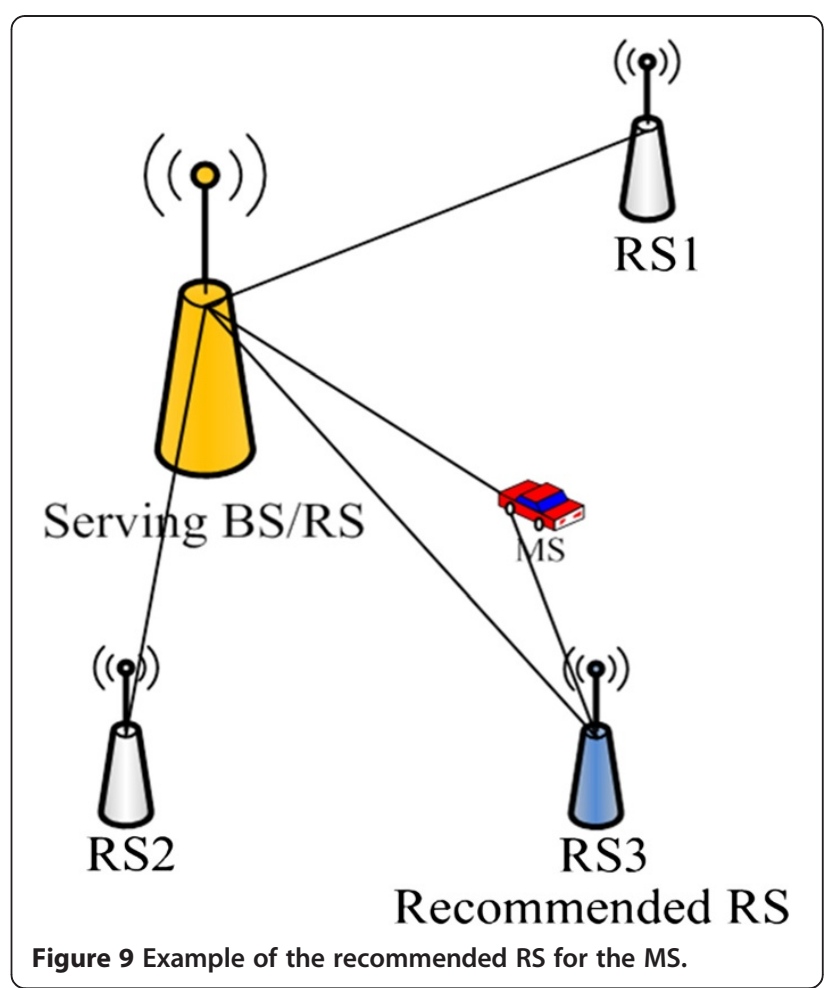

and sends the information about the neighbor BSs or RSs to the MS in IEEE 802.16j systems. An MS cannot receive data packets from the serving BS/RS during the RSS measurement for the neighbor BSs and RSs. The RSS measurement overhead increases because an MS should measure the RSS of the neighbor BSs and RSs. Therefore, the network systems need more time to handle the scanning BSs and RSs when the number of BS and RS increases. It results in more overhead in the handover scanning procedure. In our proposed scheme, the measurement function is also periodically calculated by the BS and sends the information about the

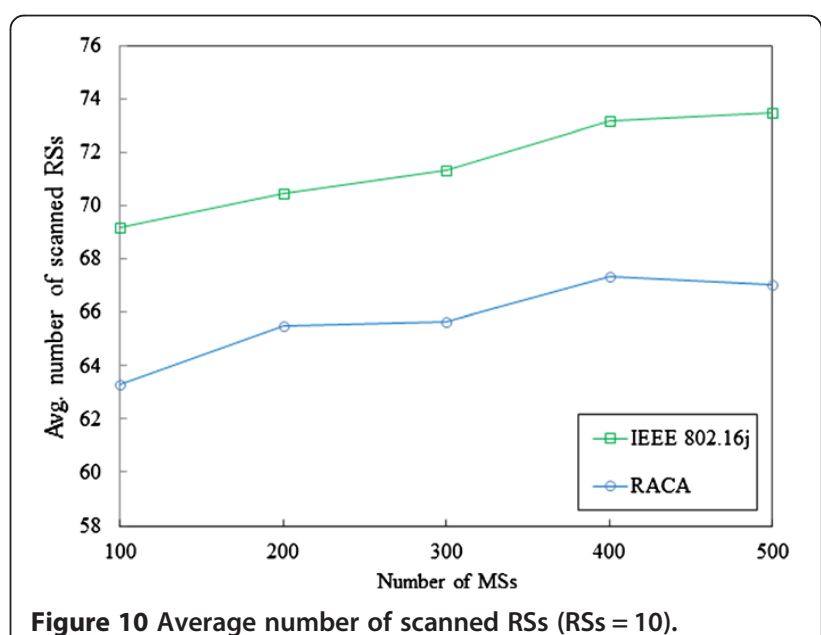




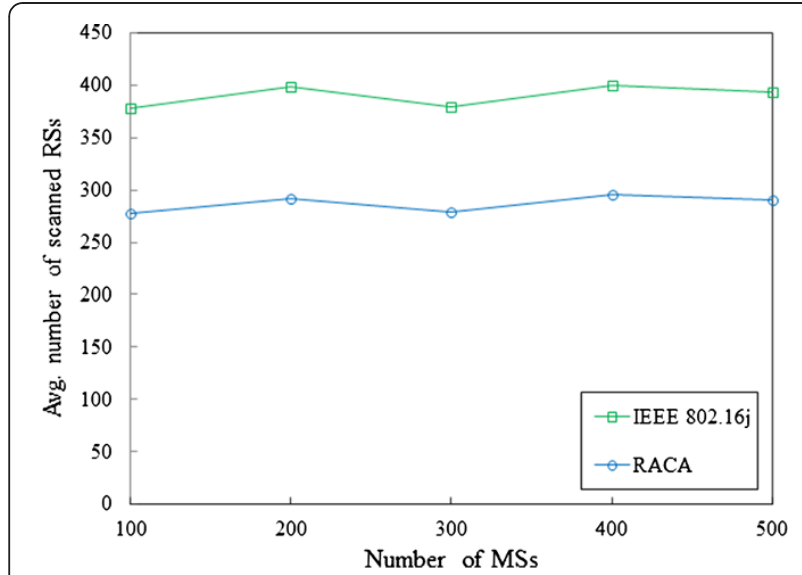

Figure 11 Average number of scanned RSs (RSs $=30$ ).

recommended RS to the MS for scanning. We eliminate the unnecessary associations and scanning intervals, and reduce the handover scanning procedure efficiently. The required computing time for handling the MS to switch the current serving BS/RS to the new BS/RS is also reduced. Based on the moving behavior of the MS, the proposed scheme makes an adaptive decision for dealing with the handover process.

\section{Performance analysis}

In this section, we evaluate the performance of our proposed RACA scheme using a simulation model. We describe our simulation model and illustrate the simulation results, comparing our scheme with IEEE 802.16j standard scheme. We design a simulation environment by using C\#. The simulation follows the transparent relay frame structure [2] and there is no intracell interference. We assume that the relay link between the BS and the RS is in line-of-sight (LOS), while the access links between the BS and the MS and between the RS and the

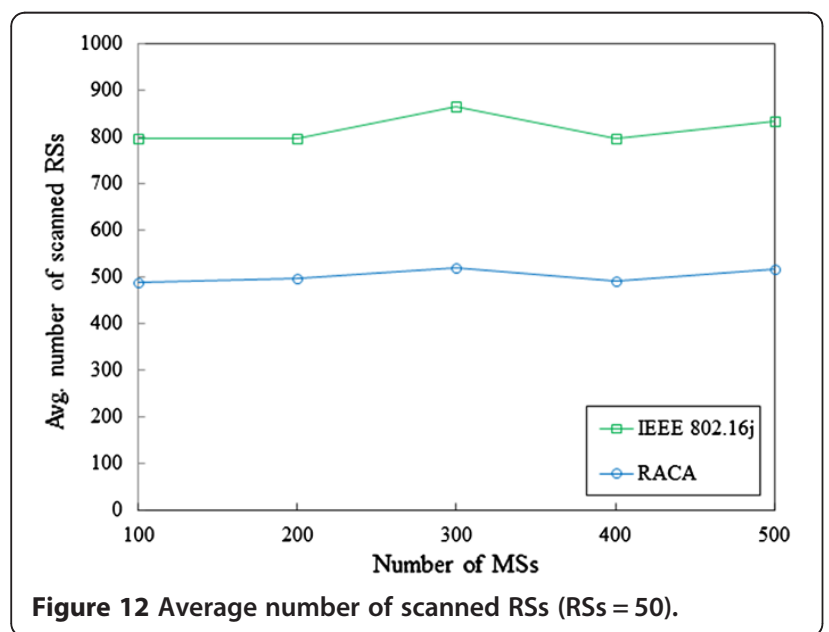

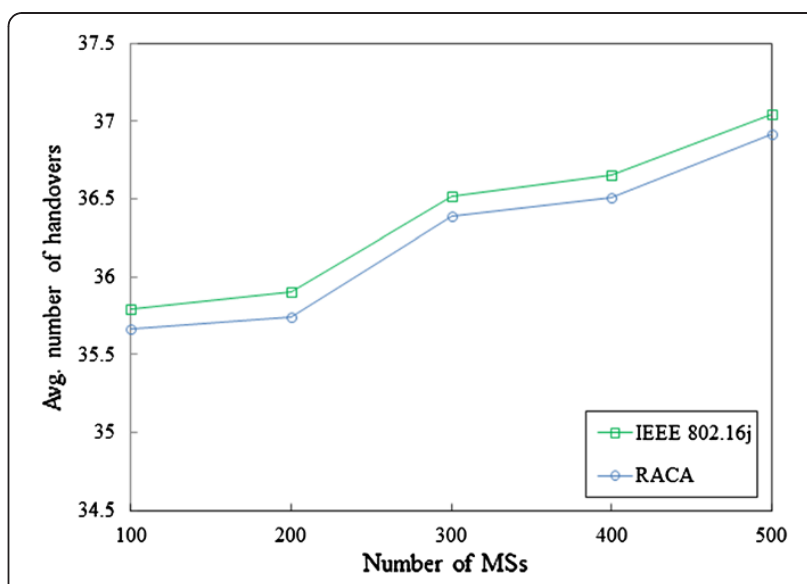

Figure 13 Average number of handovers (RSs $=10$ ).

MS are in non-LOS. The suburban macrocellular environment is assumed. The assumptions for our simulation study are as follows:

The simulation environment is composed of 100 cells and a BS is fixed and located at the center of each cell. The coverage radius of the BS is $5 \mathrm{~km}$.

The positions of the RSs are randomly distributed in the cell. For each cell, the number of RSs is equal.

The coverage radius of the RS is $1 \mathrm{~km}$. The numbers of RSs are 10, 30, and 50. The RSs are determined by RS deployment strategy which is out of the scope of this article.

The location of each MS is randomly distributed in each cell at the initial state. The numbers of MSs are 100, 200, 300, 400, and 500.

The speed of each MS is uniformly distributed between 0 and $120 \mathrm{~km} / \mathrm{h}$. The moving situation of each MS is random movement within the geographic area.

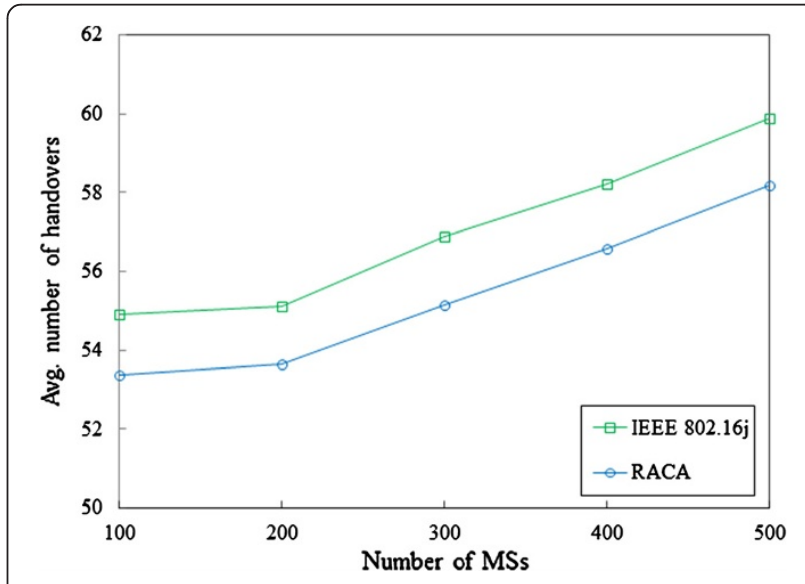

Figure 14 Average number of handovers (RSs $=30$ ). 


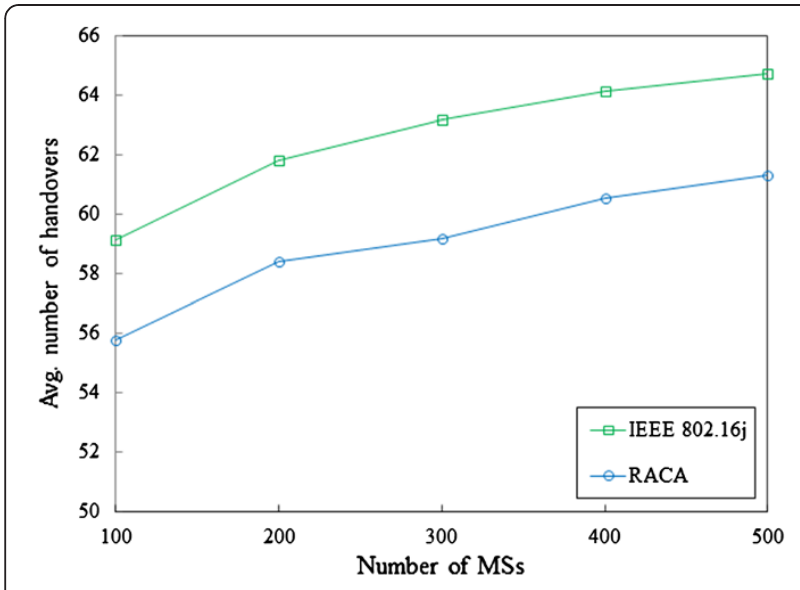

Figure 15 Average number of handovers $(\mathrm{RSs}=50)$.

The average time to measure the RSS for a BS or RS is $5 \mathrm{~ms}$ and the average time for the handover decision phase is $110 \mathrm{~ms}$ [16].

The distances between MS and serving BS/RS and the RSs are estimated based on the coding rate and receiver SNR as shown in Table 2.

Performance measures obtained on the basis of ten simulation runs are plotted as a function of the average number of scanned RSs, the average number of handovers, and the average message delay. The average number of scanned RSs is defined as the average number of scanned RSs for all MSs during the simulation. Let $n s_{m, t}$ denote the number of scanned RSs of the $m$ th MS at time $t$. If there are $M$ MSs in the system, the average number of scanned RSs can be calculated by

$$
\text { Average number of scanned RSs }=\frac{1}{M} \sum_{m=1}^{M} \sum_{t=t_{s}}^{t_{f}} n s_{m, t}
$$

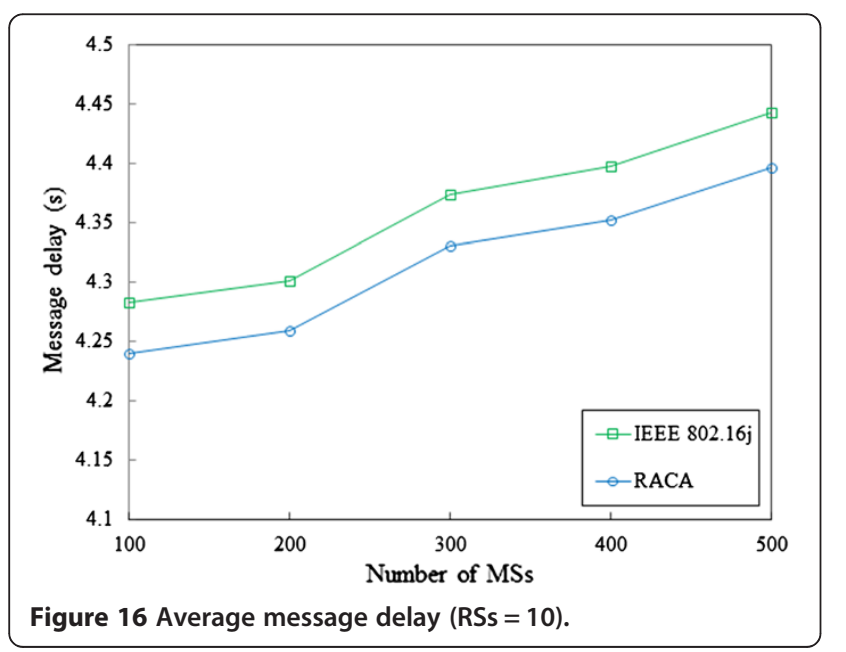

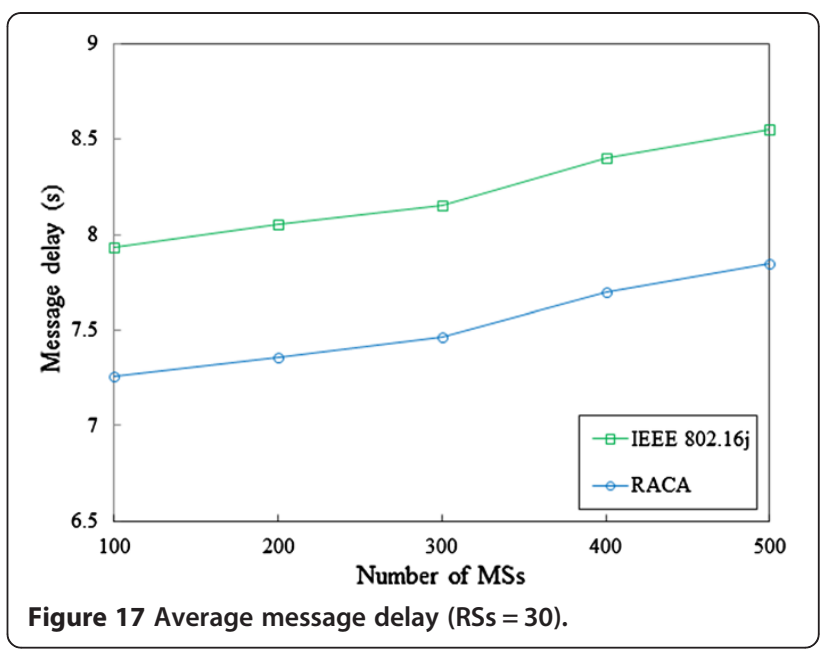

where $t$ is the simulation time from $t_{s}$ to $t_{f}$. The average number of handovers is defined as the average number of handovers for all MSs during the simulation. The message delay is defined as the average message day time for all MSs in the handover process during the simulation. Let $n h_{m, t}$ and $m_{d m, t}$, respectively, be the number of handovers and message delay time of the $m$ th MS at time $t$. If there are $M$ MSs in the system, the average number of handovers and the message delay can be calculated by

$$
\begin{aligned}
& \text { Average number of handovers }=\frac{1}{M} \sum_{m=1}^{M} \sum_{t=t_{s}}^{t_{f}} n h_{m, t} \\
& \text { Message delay }=\frac{1}{M} \sum_{m=1}^{M} \sum_{t=t_{s}}^{t_{f}} m d_{m, t}
\end{aligned}
$$

Figures 10, 11, and 12 show the average number of scanned RSs when the number of MSs varies from 100 to 500 and the number of RSs in each cell is 10,30 , and

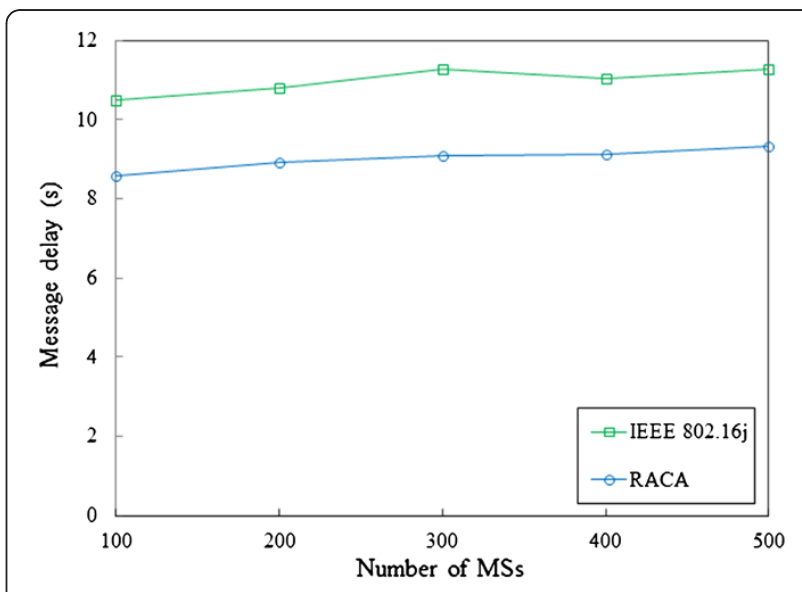

Figure 18 Average message delay (RSs $=50$ ). 
50 , respectively. It is observed that the average number of scanned RSs of IEEE 802.16j standard scheme is higher than that of our proposed scheme. According to Figures 10, 11, and 12, it is evident that different numbers of RSs in the cell result in different numbers of scanned RSs for the MSs. For the three figures described above, the total number of scanned RSs of IEEE 802.16j standard scheme is approximately 426. However, the proposed scheme achieves a total average number of scanned RSs that is approximately 285. This is because too many unnecessary scanning of the target BSs or RSs result in more number of scanned RSs for the MSs in the IEEE 802.16j standard scheme. It means that the network systems need more time to negotiate the association parameters and to handle scanning the BSs and the RSs in the handover process. In contrast, our proposed scheme estimates the potential moving path of the MS by using the RACA, which reduces the unnecessary scanning of the target BSs or RSs efficiently.

Figures 13, 14, and 15 show the average number of handovers when the number of MSs varies from 100 to 500 and the number of RSs in each cell is 10, 30, and 50, respectively. It is observed that the average number of handovers increases with an increase in the number of MSs. The average number of handovers of the two schemes exhibit similar trends. However, the average number of handovers for IEEE 802.16j standard scheme increases noticeably when the number of RSs in each cell increases. Due to the improved handover process approach in our proposed scheme, the average number of handovers of our proposed scheme is less than that of IEEE 802.16j standard scheme over various numbers of MSs. For example, when the number of RSs in the cell is 50 and the number of MSs is 500 , the proposed scheme achieves the average number of handovers that is approximately 3.5 lower than that of IEEE 802.16j standard scheme. The reason for this behavior is that the suitable target BS or RS can be recommended by using the proposed algorithm. It results in a lower average number of handovers.

Figures 16, 17, and 18 show the average message delay when the number of MSs varies from 100 to 500 and the number of RSs in each cell is 10,30 , and 50 , respectively. It is evident that the average message delay of our propose scheme is lower than that of IEEE 802.16j standard scheme. From the simulation results, it is clear that our proposed scheme achieves superior performance for varying number of MSs in the handover process for IEEE 802.16j multi-hop relay network systems. The main reason is that the proposed scheme makes an adaptive decision for handling the handover process based on the moving behavior of the MS, thus can effectively deal with the sudden traffic surges. The handover process can be accelerated. Therefore, the message delay time caused by transmission interruption can be reduced. This shows that our proposed scheme is promising and more suitable than IEEE 802.16j standard scheme in a multi-hop relay network system.

\section{Conclusions}

Handover process is one of the important components for QoS sensitive wireless networks. In order to accelerate the handover process and reduce the transmission interruption, efficient handover scanning procedure schemes and corresponding algorithms must be developed in an IEEE 802.16j multi-hop relay network system. In this article, a novel RACA is proposed to estimate the potential moving path of the MS by taking into account the moving behavior of the MS, and the distances among the MS, the RS, and the BS. The management information overhead can be reduced and the handover process can be accelerated. Simulation results indicate that our proposed algorithm achieves the low handover overhead and the low message delay in the IEEE 802.16j multi-hop relay network systems.

\section{Competing interests}

The authors declare that they have no competing interests.

Received: 30 June 2012 Accepted: 31 January 2013

Published: 21 February 2013

\section{References}

1. IEEE Std, 802.16-2009, IEEE Standard for Local and metropolitan area networks, Part 16: Air Interface for Broadband Wireless Access Systems (2009)

2. IEEE Standard for Local and metropolitan area networks, Part 16: Air Interface for Broadband Wireless Access Systems, Amendment for Multihop Relay Specification. (2009)

3. S Ann, KG Lee, HS Kim, A path selection method in IEEE 802.16j mobile multi-hop relay networks, in Proceedings of the IEEE Second International Conference on Sensor Technologies and Applications, (Cap Esterel, France, 2008), pp. 808-812

4. Y Ge, S Wen, Y-H Ang, Analysis of optimal relay selection in 802.16 multihop relay networks, in Proceedings of the IEEE Wireless Communications and Networking Conference (WCNC), (Budapest, Hungary, 2009), pp. 1-6

5. Y Ge, S Wen, Y-H Ang, Y-C Liang, Optimal relay selection in 802.16j multihop relay vehicular networks. IEEE Trans. Veh. Technol 59(5), 2198-2206 (2010)

6. S-S Wang, H-C Yin, Y-H Tsai, S-T Sheu, An effective path selection metric for IEEE 802.16-based multi-hop relay networks, in Proceedings of the IEEE Symposium on Computers and Communications (ISCC), (Aveiro, Portugal, 2007), pp. 1051-1056

7. KP Shih, SS Wang, HC Yin, A high spectral efficiency and load-aware metric for path selection in IEEE 802.16j multi-hop relay networks, in Proceedings of the IEEE Symposium on Computers and Communications (ISCC), (Sousse, Tunisia, 2009), pp. 61-66

8. S Cho, J Kwun, C Park, JH Cheon, OS Lee, K Kim, Hard handoff scheme exploiting uplink and downlink signals in IEEE 802.16e systems, in Proceedings of the IEEE 63rd Int. Conf. Vehicular Technology (VTC 2006-Spring), (Melbourne, Australia, 2006), pp. 1236-1240

9. SH Lee, Y Han, A novel inter-FA handover scheme for load balancing in IEEE 802.16e system, in Proceedings of the IEEE 63rd Int. Conf. Vehicular Technology (VTC 2007-Spring), (Dublin, Ireland, 2007), pp. 763-767

10. $\mathrm{PH}$ Tseng, KT Feng, A predictive movement based handover algorithm for broadband wireless networks, in Proceedings of the IEEE Wireless Communications and Networking Conference (WCNC), (Las Vegas, Nevada, USA, 2008), pp. 2834-2839

11. J Park, S Oh, J Jeong, H Choo, Fast handover scheme based on mobile locations for IEEE 802.16e networks, in Proceedings of the International 
Conference on Computational Science and Its Applications, (Yongin, Korea, 2009), pp. 62-67

12. H Fehri, J Chitizadeh, MH Yaghmaee, A novel downlink handover priority scheduling algorithm for providing seamless mobility and QoS in IEEE 802.16e BWA system, in Proceedings of the International Conference on Communications and Mobile Computing, 3rd edn. (Kunming, Yunnan, China, 2009), pp. 227-231

13. R Ellouze, M Gueroui, MA Alimi, Optimising handover for real-time flows in mobile wimax network, in Proceedings of the IEEE Symposium on Computers and Communications (ISCC), (Sousse, Tunisia, 2009), pp. 40-45

14. JH Park, KY Han, DH Cho, Reducing inter-cell handover events based on cell ID information in multi-hop relay systems, in Proceedings of the IEEE 65th Vehicular Technology Conference (VTC2007-Spring), (Dublin, Ireland, 2007), pp. 743-747

15. Z Becvar, P Mach, R Bestak, Optimization of handover scanning procedure in WiMAX networks with relay stations, in Proceedings of the $3 \mathrm{rd}$ International Symposium on Wireless Pervasive Computing (ISWPC 2008), (Santorini, Greece, 2008), pp. 581-585

16. S Cho, EW Jang, JM Cioffi, Handover in multihop cellular networks. IEEE Commun. Mag. 47, 64-73 (2009)

17. L Yun, W Ying, Z Weiliang, Y Xiaohu, A fast handover scheme in IEEE 802.16 relay networks, in Proceedings of the Second International Conference on Future Networks (ICFN '10), (Sanya, Hainan, China, 2010), pp. 243-247

18. J Sultan, M Ismail, N Misran, Downlink performance of handover techniques for IEEE 802.16j multi-hop relay networks, in Proceedings of the 4th IEEE/IFIP International Conference on Internet, (Tashkent, Uzbekistan, 2008), pp. 1-4

19. J Sultan, N Misran, M Ismail, MT Islam, Topology-aware macro diversity handover technique for IEEE 802.16j multi-hop cellular networks. IET Commun. 1(5), 700-708 (2011)

doi:10.1186/1687-1499-2013-45

Cite this article as: Chang: An accelerating handover process scheme for IEEE 802.16j multi-hop relay networks. EURASIP Journal on Wireless Communications and Networking 2013 2013:45.

\section{Submit your manuscript to a SpringerOpen ${ }^{\circ}$ journal and benefit from:}

- Convenient online submission

- Rigorous peer review

- Immediate publication on acceptance

- Open access: articles freely available online

- High visibility within the field

- Retaining the copyright to your article

Submit your next manuscript at $\gg$ springeropen.com 